\title{
Protective Effects of Vitamin E Consumption against 3MT Electromagnetic Field Effects on Oxidative Parameters in Substantia Nigra in Rats
}

\author{
Ahmad Ali Ghanbari ${ }^{1 *}$, Kobra Shabani ${ }^{1}$, Daryoush Mohammad Nejad ${ }^{2}$
}

1. Department of Anatomy, School of Medicine, Tabriz University of Medical Sciences, Tabriz, Iran

2. Drug Applied Research Center, Medical Research and Development Complex, Department of Anatomy, Tabriz University of Medical Sciences, Tabriz, Iran

Citation: Ghanbari, A. A., Shabani, K., \& Mohammad Nejad, D. (2016). Protective effects of vitamin E consumption against 3MT electromagnetic field effects on oxidative parameters in substantia nigra in rats. Basic and Clinical Neuroscience, 7(4), 315-322. http://dx.crossref. org/10.15412/J.BCN.03070404

: http://dx.crossref.org/10.15412/J.BCN.03070404

Article info:

Received: 11 March 2016

First Revision: 30 March 2016

Accepted: 7 May 2016
Key Words:

Electromagneric field, Lipid peroxidation, GPX MDA, SOD, CAT

\begin{abstract}
A B S T RA C T
Introduction: Electromagnetic fields (EMFs) can influence the biological system by the formation of free radicals in cells. The EMFs are able to deteriorate defense system against free radicals that leads to oxidative stress (OS). Lipid peroxidation process (LPO) is an index of oxidative stress, and the Malandialdehyde (MDA) is the final product of LPO. Vitamin E is the most important antioxidant which inhibits the LPO process. The aim of this study was to evaluate the effects of 3MT EMF exposure on oxidative stress parameters in substantia nigra and the role of vitamin $\mathrm{E}$ in reducing oxidative stress and preventing of LPO process.
\end{abstract}

Methods: 40 male Wistar rats were randomly divided into 4 groups: 1) Control group: received standard food without exposure to EMF and without consumption of vitamin E, 2) Experimental group 1: was exposed to EMF (3MT) $4 \mathrm{~h} /$ day for 50 days, 3) The experimental group 2: received $200 \mathrm{mg} / \mathrm{kg}$ vitamin E with gavage every day and also was exposed to EMF (3MT) $4 \mathrm{~h} /$ day for 50 days, 4) Sham group: received water with gavage for 50 days.

Results: A significant increase in MDA levels and Glutation peroxidase (GSH-Px) activity of the substantia nigra following 50 days exposure to EMF was detected, but the superoxide dismutase (SOD) activity was decreased. Exposure did not change total antioxidant capacity (TAC) levels in plasma. Vitamin E treatment significantly prevented the increase of the MDA levels and GSHPx activity and also prevented the decrease of SOD activity in tissue but did not alter TAC levels. The GSH-Px activity increased because the duration and intensity of exposure were not enough to decrease it.

Conclusion: We demonstrated two important findings; that 50 days exposure to 3 MT electromagnetic field caused oxidative stress by increasing the levels of MDA, and decreasing SOD activity in the substantia nigra; and that treatment with the vitamin E significantly prevented the oxidative stress and lipid peroxidation.

* Corresponding Author:

Ahmad Ali Ghanbari, PhD

Address: Department of Anatomy, School of Medicine, Tabriz University of Medical Sciences, Tabriz, Iran

Tel: +98 (914) 412950

E-mail: ghanbaria34@gmail.com 


\section{Introduction}

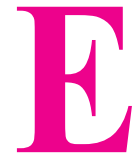

lectromagnetic field (EMF) is a physical field that is produced by charged particles (Hamidian Jahromi et al., 2014). Human use of devices that produce EMF, such as television, computers, mobile phone, kitchen appliance, and so on, may be harmful for their lives. EMFs are produced not only by technological appliances but also are used in medical diagnostic and therapeutic devices such as magnetic resonance imaging (MRI) and microwave imaging (Consales, Merla, Marino, \& Benassi, 2012). The use of EMF producing appliances and medical diagnostic and therapeutic equipment causes daily exposure to EMF. For these reasons, research on the effects of EMF on biological system is one of the important subjects that has been considered by investigators (Hamidian Jahromi et al., 2014). EMFs influence metabolic processes in human body and have some biological effects on living cell by several mechanisms. Because of energy that EMF carries, it has effect on chemical bonds between adjacent atoms, elevates the energy level of them and eventually produce molecules or atoms called free radicals (Rollwitz, Lupke, \& Simkó, 2004).

In addition to this, EMF can extend the lifetime of free radicals that were previously present in the cells, and therefore increase the concentration of free radicals in cell (Lee et al., 2004; Yokus, Cakir, Akdag, Sert, \& Mete, 2005). Free radicals are defined as molecules or atoms or ions that have unpaired electron in their outer orbit (Goraca, Ciejka, \& Piechota, 2010). Therefore, they are chemically very reactive and can react with another molecules or even with themselves (Consales et al., 2012). Free radicals are divided in two species: reactive oxygen species (ROS) and reactive nitrogen species (RNS) (Canseven, Coskun, \& Seyhan, 2008; Goraca et al., 2010). In addition, EMF exposure can disarrange the cellular balance by producing reactive oxygen species. Increased concentration of ROS is harmful for cells (Consales et al., 2012). There is defense system in living organism that act against the different oxidants and protect cells against oxidative damage (Goraca et al., 2010).

This system has two types of antioxidants that include vitamin antioxidants (A, C, and E) as well as enzymatic antioxidants such as catalase (CAT) and glutation peroxidase (GSH-Px) and superoxide dismutase (SOD) (Goraca et al., 2010). The brain is almost lack of catalase (CAT) enzyme (Cui, Luo, Xu, \& Murthy, 2004). Superoxide radical $\left(\mathrm{O}_{2}^{-}\right)$is very toxic and SOD rapidly converts superoxide radical $\left(\mathrm{O}_{2}^{-}\right)$into hydrogen peroxide $\left(\mathrm{H}_{2} \mathrm{O}_{2}\right)$ and oxygen. Hydrogen peroxide $\left(\mathrm{H}_{2} \mathrm{O}_{2}\right)$ is imme- diately destroyed by CAT and GSH-Px (Awad \& Hassan, 2008). EMFs amplify oxidative stress and can specially deteriorate all this antioxidant's defense system (Canseven et al., 2008; Goraca et al., 2010; Lee et al., 2004; McCord, 2000; Yokus et al., 2005). Since post-mitotic cells in CNS such as neurons and glial cells are susceptible to free radicals' attack, ROS has harmful effects on these cells, and therefore leads to neuronal damage (GilgunSherki, Melamed, \& Offen, 2001). Programmed cell death is one effect of oxidative damage of ROS in human cells (Salganik, 2001). Unbalance between the production of ROS, such as superoxide $\left(\mathrm{O}_{2}^{-}\right)$, hydrogen peroxide $\left(\mathrm{H}_{2} \mathrm{O}_{2}\right)$, hydroxyl $(\mathrm{OH})$, and peroxyl radical (LOO), and cells' ability for scavenging various antioxidants is defined as oxidative stress (OS) (Gutteridge, 1995).

Overproduction of ROS develops oxidative damage to biomolecules such as lipids and proteins and nucleic acids, and therefore makes changes in enzyme activity and gene expression eventually leading to many diseases, for example, arthrosclerosis, diabetes, rheumatoid arthritis, cardiovascular disease, stroke, and neurodegenerative disease (Fang, Yang, \& Wu, 2002; Fridovich, 1999; Mattson, 2004). The most important index of oxidative stress is degeneration of polyunsaturated fatty acids (PUFA) in organelles and cytoplasmic membrane of the cells through reaction of free radicals with PUFA in membrane. Final production of LPO is Malandialdehyde (MDA) (Canseven et al., 2008; Goraca et al., 2010). MDA is a toxic molecule, and it is marker of lipid peroxidation and oxidative stress in body tissues (Köylü, Mollaoglu, Ozguner, Nazýroölu, \& Delibap, 2006). For some reasons, the brain is susceptible to oxidative stress. The brain consumes the highest amount of oxygen in the human body and, although most of oxygen is converted into $\mathrm{CO}_{2}$ and water., Small amount of this oxygen produces ROS (Nazıroğlu, 2007) and brain tissue is rich in polyunsaturated fatty acids that are ROS target, and in addition to these, the metabolism rate in brain is high (Irmak, Oztas, Yagmurca, Fadillioglu, \& Bakir, 2003; Özmen et al., 2007; Salford, Brun, Eberhardt, Malmgren, \& Persson, 2003).

Lipid peroxidation maintains to undermine the substrate until a chain breaking antioxidant, for instance vitamin E, enters the reaction and stops continuing the chain reaction of LPO (Gutteridge, 1995). Vitamin E is a lipid soluble antioxidant that inhibits peroxidation of PUFA and protects membranes from destructive effects of free radicals (Tappel, 1972). Parkinson's disease is a progressive neurodegeneration disease that is characterized by the death of dopamine-generating cells in the substantia nigra (Fariss \& Zhang, 2003). Oxidative stress is considered one of pathogenic factors in Parkinson's disease (Mattson, 
2004). Recent findings are suggested potential correlation between Parkinson's disease and EMF (Adey, 1993; Johansen, 2000; Savitz, Checkoway, \& Loomis, 1998). There is no experimental study of possible harmful effects of EMF exposure on substantia nigra cells degeneration; Therefore, the current study investigated the effects of EMF exposure on MDA levels and activity of antioxidant enzymes (SOD, GSH-Px) in substantia nigra and levels of TAC in plasma and also investigated the effects of vitamin $\mathrm{E}$ in inhibiting the alteration of these parameters.

\section{Methods}

40 Male Wistar rats weighing 160-180 grams were used as experimental animals in this study. They were kept under standard condition with free access to standard laboratory water and food. They were housed in polypropylene cages in same room. The room had 12-12 hours light dark cycle. The protocol and study method was approved by the local animal Care Committee.

In order to produce electromagnetic field (EMF), a generator using $220 \mathrm{~V}$ and $50 \mathrm{~Hz}$ electricity was used. The generator produced an electromagnetic field with 3 MT intensity. The main part of the EMF producing device was made of two cupric coils that were based on Helmholtz coil. The direction of electromagnetic field produced by coils was opposite to one another and due to this manner; the device generated a homogenous electromagnetic field in the center of the device where the cages of animals were inhabited in. The device was designed to accommodate 3 cages with capacity for 7 rats per cage. The direction of the field produced by a coil can be determined by the right hand grip rule. The device was also equipped with a transformer to control the electrical current and an appropriate fan to prevent the increase in animal internal temperature. In addition to this, the intensity of EMF produced by the device was examined before and during the study using the device-associated Tesla meter.

The animals were randomly divided into four groups with 10 rats in each group: 1$)$ Control group $(n=10)$ : received standard food without being exposed to EMF and without consumption of vitamin E, 2) Experimental group $1(n=10)$ : received their food similar to the control group but was exposed to EMF (3MT) 4 hours a day for 50 days, 3) Experimental group 2 ( $\mathrm{n}=10)$ : received $200 \mathrm{mg} /$ $\mathrm{kg}$ vitamin E per day with gavage and also was exposed to EMF (3MT) (4 hours a day for 50 days, 4) Sham group $(n=10)$ : received water with gavage every day for 50 days.

Immediately after exposure period, rats were anesthetized by placing them in a glass jar containing cot- ton dipped in chloroform. Animals were sacrificed and under direction of stereomicroscope, the midbrain part of the brain stem was dissected and samples of their substantia nigra were collected. Also 4cc of their Venus blood samples was collected, then the serum of them was separated with centrifuge.

Malondialdehyde assessment: MDA levels were assayed by method described as Tiobarbitoric acid reactive substance (TBARS) (Somi et al., 2009). In this method MDA level was measured based on reaction with tiobarbitoric acid. The extraction was performed with normal butanol and assessment was done using spectrophotometer and absorption were compared with standard curve (Pezeshkian et al., 2011).

Glutathione peroxidase and superoxide dismutase activity assessment: To assay theses enzymes activity, samples were homogenized in $1 / 15 \% \mathrm{KCL}$ solution (Somi et al., 2009).

\subsection{GSH-Px activity assessment}

GSH-Px activity was measured by the method of Paglia and Valentine using Randox (United Kingdom) (Kerman \& Senol, 2012; Somi et al., 2009). GSH-Px catalyses the oxidation of glutathione (GSH) by Cumen hydroperoxide. In the presence of glutathione reductase (GR) and NADPH, the oxidized glutathione (GSSG) is immediately converted to the reduced form with a concomitant oxidation of NADPH to $\mathrm{NADP}^{+}$. The decrease in absorbance at $340 \mathrm{~nm}$ is measured (according to Randox kit protocol).

\subsection{SOD activity assessment}

The role of superoxide dismutase (SOD) is to accelerate the dismutation of the toxic superoxide radical (02) to hydrogen peroxide and molecular oxygen. SOD was measured using Randox (United Kingdom). The method for assaying this enzyme employs xanthine and xanthine oxidase (XOD) to produce superoxide radicals which react with 2-(4-iodophenyl)-3-(4-nitrophenol)-5-phenyltetrazolium chloride (I.N.T.) to form a red formazan dye. The superoxide dismutase activity is then measured by the degree of inhibition of this reaction (Somi et al., 2009).

\subsection{Determination of TAC concentration in plasma}

ABTS (2, 2-Azino-di-[3-ethylbenzthiazoline sulphonate]) is incubated with a peroxidase (metmyoglobin) and $\mathrm{H}_{2} \mathrm{O}_{2}$ to produce the radical cation ABTS. This has a relatively stable blue-green color, which is measured 
at $600 \mathrm{~nm}$. Antioxidants in the added sample cause suppression of this color production to a degree which is proportional to their concentration (according to Randox kit protocol).

\subsection{Statistical analysis}

The data analysis was performed using the SPSS-16 package. ANOVA test was used to compare between the means of the different parameters of the four studied groups that followed by post hoc analysis with Tukey test. P-value lower than 0.05 was considered significant.

\section{Results}

The effect of 50 days exposure to 3 MT electromagnetic field and treatment with vitamin $\mathrm{E}$ on oxidative parameters in substantia nigra are depicted in Table 1 and figures. As shown in Table 1 and Figure 1, in the exposed group after 50 days exposure to EMF, MDA levels $(0.28 \pm 0.03$ $\mathrm{nmol} / \mathrm{mg}$ protein) were significantly increased in substantia nigra when compared to control $(0.18 \pm 0.06 \mathrm{nmol} /$ $\mathrm{mg}$ protein) and sham $(0.19 \pm 0.04 \mathrm{nmol} / \mathrm{mg}$ protein $)$ groups $(\mathrm{P}<0.05, \mathrm{~F}=9.214)$. Whereas the increase in MDA levels is an indicator of lipid peroxidation, this results revealed that 50 days exposure to EMF can increase lipid peroxidation process in substantia nigra. No significant difference in MDA levels was noted between the EMF exposure plus vitamin E group $(0.15 \pm 0.03 \mathrm{nmol} / \mathrm{mg}$ protein) and control group ( $\mathrm{P}>0.05)$. In other words, 50 days exposure to EMF induced significant increase in MDA levels and Vitamin E treatment significantly prevented the increase in the MDA levels of the substantia nigra $(\mathrm{P}<0.05, \mathrm{~F}=9.214)$. MDA levels were not different between the control and sham groups $(\mathrm{P}>0.05)$.

As shown in Table 1 and Figure 2, a significant decrease was detected in SOD activity in the exposed group after 50 days exposure to EMF $(2.30 \pm 0.05 \mathrm{u} /$ $\mathrm{mg}$ protein) in comparison with control $(2.58 \pm 0.13 \mathrm{u} /$

Table 1. SOD, GSH-Px activity and levels of MDA in substantia nigra, and TAC levels in plasma for each groups.

\begin{tabular}{|c|c|c|c|c|}
\hline \multirow{2}{*}{ Group } & MDA & SOD & GSH-Px & TAC \\
\cline { 2 - 5 } & nmol/mg Protein & u/mg Protein & u/mg Protein & $0.62 \pm 0.05$ \\
\hline Control & $0.18 \pm 0.06$ & $2.58 \pm 0.13$ & $0.49 \pm 0.05$ & $0.64 \pm 0.06$ \\
\hline EMF & $0.28 \pm 0.03^{*}$ & $2.30 \pm 0.05^{*}$ & $0.68 \pm 0.08^{*}$ & $0.68 \pm 0.05$ \\
\hline EMF+Vitamin E & $0.15 \pm 0.03^{+}$ & $2.64 \pm 0.27^{+}$ & $0.54 \pm 0.10^{+}$ & $0.64 \pm 0.06$ \\
\hline Sham & $0.19 \pm 0.04$ & $2.54 \pm 0.14$ & $0.49 \pm 0.05$ & \\
\hline
\end{tabular}

${ }^{*} \mathrm{P}<0.05$ shows significant difference in comparison with the control group; ${ }^{+} \mathrm{P}<0.05$ shows significant difference in comparison with EMF group. Values are given as mean \pm SD.

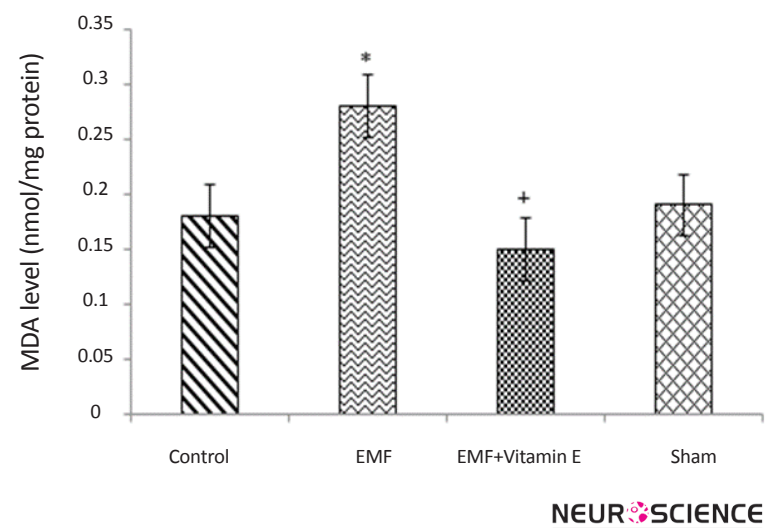

Figure 1. MDA levels in substantia nigra of rats for each groups. ${ }^{*} \mathrm{P}<0.05$ shows significant difference in comparison with the control group; ${ }^{+} \mathrm{P}<0.05$ shows significant difference in comparison with EMF group. Results are expressed as mean \pm SD of MDA levels. Statistical analysis was carried out with one-way ANOVA $(\mathrm{F}=9.214)$.

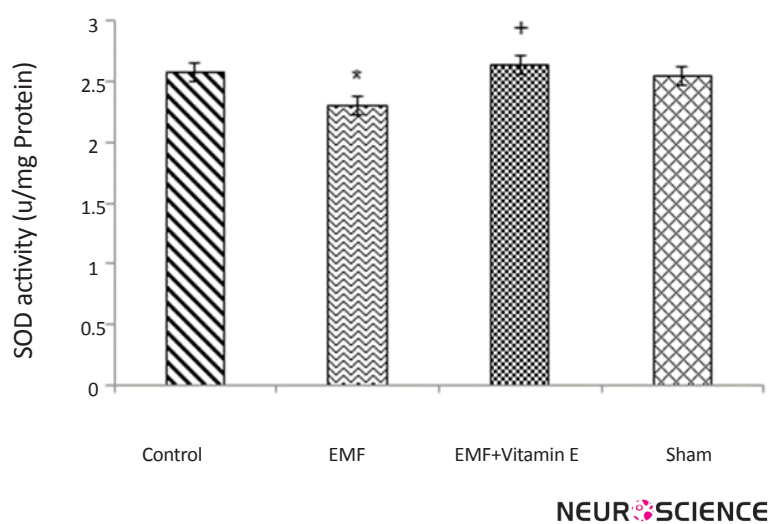

Figure 2. SOD activity in substantia nigra of rats for each groups. ${ }^{*} \mathrm{P}<0.05$ shows significant difference in comparison with the control group; ${ }^{+} \mathrm{P}<0.05$ shows significant difference in comparison with EMF group. Results are expressed as mean \pm SD of SOD activity. Statistical analysis was carried out with one-way ANOVA ( $\mathrm{F}=5.325)$. 


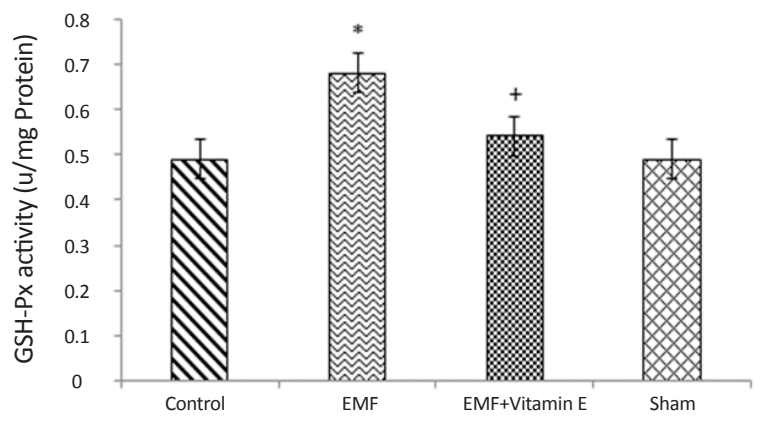

NEUR :SCIENCE

Figure 3. GSH-Px activity in substantia nigra of rats for each groups. ${ }^{*} \mathrm{P}<0.05$ shows significant difference in comparison with the control group; ${ }^{+} \mathrm{P}<0.05$ shows significant difference in comparison with EMF group. Results are expressed as mean \pm SD of GSH-Px activity. Statistical analysis was carried out with one-way ANOVA ( $\mathrm{F}=9.231)$.

$\mathrm{mg}$ protein)and sham $(2.54 \pm 0.14 \mathrm{u} / \mathrm{mg}$ protein) groups $(\mathrm{P}<0.05, \mathrm{~F}=5.325)$. ROS production may be the result of the observed reduction in the SOD activity, and our results revealed that EMF exposure can increase oxidative stress in substantia nigra. SOD activity did not show statistical difference between control group and sham group $(\mathrm{P}>0.05)$. The decrease in SOD activity significantly prevented by vitamin $\mathrm{E}$ treatment $(2.64 \pm 0.27 \mathrm{u} / \mathrm{mg}$ protein) $(\mathrm{P}<0.05, \mathrm{~F}=5.325)$ because there was no significant alteration in SOD activity between the EMF exposure plus vitamin E group and control group $(\mathrm{P}>0.05)$.

Table 1 and Figure 3 show that electromagnetic field produced a significant increase in GSH-Px activity in substantia nigra $(0.68 \pm 0.08 \mathrm{u} / \mathrm{mg}$ protein $)$ when compared to control $(0.49 \pm 0.05 \mathrm{u} / \mathrm{mg}$ protein $)$ and sham $(0.49 \pm 0.05 \mathrm{u} /$ $\mathrm{mg}$ protein) groups $(\mathrm{P}<0.05, \mathrm{~F}=9.231)$. GSH-Px activity increased because the duration and intensity of exposure were not enough to decrease it. Vitamin E treatment prevented the increase in GSH-Px activity in substantia nigra following 50 days exposure $(\mathrm{P}<0.05, \mathrm{~F}=9.231)$ and $\mathrm{GSH}-$ Px activity in this group was remind to be $0.54 \pm 0.10 \mathrm{u} /$ $\mathrm{mg}$ protein. GSH-Px activity was not different between control group and sham group $(\mathrm{P}>0.05)$.

TAC levels were measured in serum. As shown in Table 1 and Figure 4, there was no significant alteration in TAC levels in all groups $(\mathrm{P}>0.05)$ and 50 days exposure to EMF was not able to alter TAC levels in plasma.

\section{Discussion}

EMF exposure elevates the concentration of free radicals in cells by forming free radicals (Bediz, Baltaci,

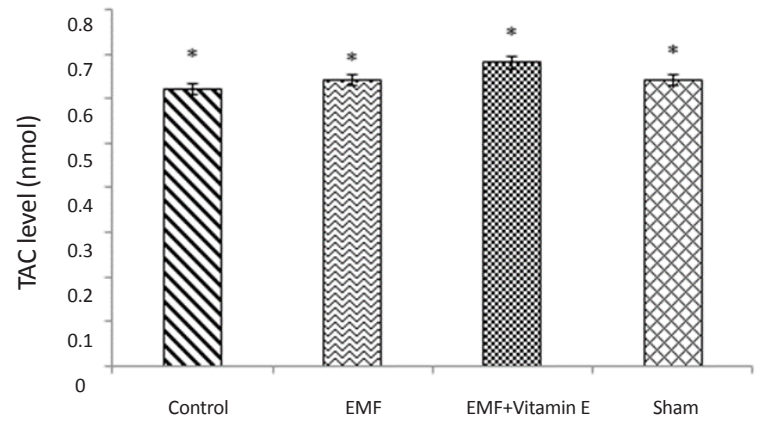

\section{NEUR:SCIENCE}

Figure 4. TAC levels in plasma of rats for each groups. ${ }^{*} \mathrm{P}>0.05$ shows that there was no statistical difference between all groups. Exposure to EMF was not able to alter TAC levels in plasma. Results are expressed as mean \pm SD of TAC levels. Statistical analysis was carried out with oneway ANOVA.

Mogulkoc, \& Öztekin, 2006) and causes increase in their life span in cells (Lee et al., 2004; Yokus et al., 2005). EMF exposure also can deteriorate antioxidant defense system against free radicals which leads to oxidative stress (OS) (Canseven et al., 2008; Goraca et al., 2010; Lee et al., 2004; McCord, 2000; Yokus et al., 2005). OS makes various damages in cells. ROS, attack cell and react with PUFA in cell membrane and leads to LPO. MDA is produced as a consequence of LPO (Canseven et al., 2008; Goraca et al., 2010). Brain tissue is sensitive to ROS attack because it has high metabolic rate as well as antioxidant defense system is poor in brain and also brain tissue is rich in PUFA (Goraca et al., 2010; (Irmak et al., 2003; Salford et al., 2003).

In this study, we have investigated protective effects of vitamin $\mathrm{E}$ against alteration of oxidative parameters in substantia nigra following 50 days exposure to EMF. A significant increase in MDA levels as an indicator of lipid peroxidation was observed in exposed group. (Coşkun, Balabanl, Canseven, \& Seyhan, 2009) investigated the effects of magnetic fields on oxidative parameters in pigs. Their results were inconsistent with our results. They reported that brain MDA levels were significantly decreased both in the continuous and the intermittent exposure groups when compared to control group. In their study, duration and time of exposure were shorter than our study. This finding suggests that duration of exposure can play an important role in side effects of EMF, for instance, lipid peroxidation and changes of antioxidant defense system. In their study, increased concentration of GSH has protected brain tissue against lipid peroxidation and ROS damages. 
In the brain tissue, high concentration of GSH shows preparation of cell against oxidative damage (Akdag et al., 2010; Ayala, Muñoz, \& Argüelles, 2014; Ilhan et al., 2004). Awad and Hassan (2008) investigated the effects of electromagnetic radiation in the brain of rats. They exposed rats to $900 \mathrm{MHz}$ EMR from mobile phone $1 \mathrm{~h} /$ day for one week. Their results were similar to our findings. Their investigation revealed that lipid peroxidation increased after exposure to mobile phone. Similar results on brain came from (Jelenković et al., 2006). They expressed that MDA levels increased in cortex and basal forebrain following 7-day exposure. There are some other studies about correlation between MDA level and EMF exposure in other organs. Moussa (2009) showed that MDA levels increased following the increase in duration of EMF exposure and Meral et al. (2007) reported an increase in MDA levels in pigs after exposure to EMF 12h/day for 30 days. Increase of MDA level can be an indicator of increased formation of ROS following exposure to EMF and shows that exposure has pathophysiological effects on various tissues.

Living cells have defense system that act against harmful effects of ROS (Awad \& Hassan, 2008; Goraca et al., 2010) and protect biological biomolecules from OS damages. In the present study, we assayed antioxidant enzyme activity following exposure to $3 \mathrm{MT}$ EMF. In this study, SOD activity decreased in substantia nigra after exposure to EMF, and GSH-Px activity significantly increased following exposure and total antioxidant (TAC) level was not significantly different between groups. Ilhan et al. (2004) reported a decrease in GSHPx and SOD activity following exposure to EMF in hippocampal neuronal tissue. Yilmaz, Uz, Yucel, Altuntas, and Ozcelik, (2004) investigated the effects of $900 \mathrm{MHz}$ EMF on liver and kidney of rats and their results were inconsistent with our results about GSH-Px activity.

In our study, the increase in MDA level and decrease in SOD activity in the exposed group are important markers that show the key role of oxidative stress in these changes .Our study shows that 50 days exposure was not sufficient for decreasing GSH-Px activity in substantia nigra, and GSH-Px activity increased to act against with ROS production and duration or intensity of exposure were not enough to decrease GSH-Px activity. Similar results came from Ayata et al. (2004) investigation. They reported that following $900 \mathrm{MHz}$ radiation exposure, levels of MDA and activity of GSH-Px increased significantly. However, SOD activity decreased significantly.

ROS production may be the result of the observed reduction in the SOD activity, and our finding suggests that
EMF exposure increases oxidative stress in substantia nigra. Ilhan et al. (2004) reported a decrease in SOD and GSH-Px activity in brain tissue after exposure to EMF when compared to control group. Akdag et al. (2010) reported that TAC level decreased in ELF 500 exposure group in comparison with ELF 100 exposure group, and this reveals that the intensity of exposure influences on oxidative stress production. Their results are in contrast with our results because in their study rats were exposed to ELF $2 \mathrm{~h}$ /day for 10 month, and the duration of exposure in their study was longer than our study, and it was enough to decrease TAC level in plasma. Lipid peroxidation process has three steps: initiation, propagation, and termination.

In the initiation step, hydroxyl radical (OH.) abstracts the hydrogen from polyunsaturated fatty acid and produces carbon-centered free radical (L.) (Ayala et al., 2014; Tappel, 1972; Van Acker, Koymans, \& Bast, 1993).

$$
\mathrm{LH}+\mathrm{OH} \longrightarrow \mathrm{L}+\mathrm{H}_{2} \mathrm{O}
$$

In propagation step, L. immediately reacts with $\mathrm{O}_{2}$ and produces Peroxyl radical (LOO) and LOO. Abstracts a hydrogen from another lipid molecules and produces new L. and Lipid hydro peroxide (LOOH.) (Ayala et al., 2014; Tappel, 1972; Van Acker et al., 1993).

$$
\begin{gathered}
\mathrm{L}_{\mathrm{O}} \longrightarrow \mathrm{LOO} \\
\mathrm{LOO}+\mathrm{LH} \longrightarrow \mathrm{LOOH}+\mathrm{L}
\end{gathered}
$$

LPO maintains until a chain breaking antioxidant specially vitamin $E$ enters to reaction and stop continuing of chain reaction of LPO (Rollwitz et al., 2004). Vitamin E gives a hydrogen atom to LOO. and produces vitamin $\mathrm{E}$ radical. Then vitamin $\mathrm{E}$ radical reacts with another LOO. and forms $\alpha$-Tocopherolquinon (Ayala et al., 2014; Van Acker et al., 1993).

$$
\begin{gathered}
\mathrm{LOO}+\alpha \mathrm{TH} \longrightarrow \mathrm{LOOH}+\alpha \mathrm{T} \\
\mathrm{LOO}+\alpha \mathrm{LT} \longrightarrow \alpha \mathrm{TQ}+\alpha T
\end{gathered}
$$

In previous studies, Oral et al. (2006) showed beneficial effects of vitamin $\mathrm{E}$ in preventing endometrial changes following exposure to $900 \mathrm{MHz}$ EMF in rats. Zhang et al. (2011) observed protective effects of vitamin E and reported that vitamin $\mathrm{E}$ consumption diminishes alteration of antioxidant capacity after mobile phone exposure in pregnant and fetal mice. Mohammadnejad, Rad, Azami, and Lotfi (2011) investigated ultrastructural changes of thymus following exposure to EMF and protective effects of vitamin $\mathrm{E}$ in the prevention of this changes. They 
observed that EMF exposure makes damages in immune system, and vitamin E consumption can prevent ultrastructural alteration in tissue.

In conclusion, our results show that 50 days exposure to 3 MT electromagnetic field causes oxidative stress by increasing MDA levels, and decreasing SOD activity and treatment with vitamin E prevents the oxidative stress and lipid peroxidation in the substantia nigra.

\section{Acknowledgments}

This work was supported by Neuroscience Research Center, Tabriz University of Medical Sciences, Tabriz, Iran. The authors are thankful to Vice Chancellor of Neuroscience Research Center, Tabriz University of Medical Sciences, for financial support.

\section{Conflict of Interest}

All authors declared no conflict of interest.

\section{References}

Adey, W. R. (1993). Biological effects of electromagnetic fields. Journal of Cellular Biochemistry, 51(4), 410-16.

Akdag, M. Z., Dasdag, S., Ulukaya, E., Uzunlar, A. K., Kurt, M. A., \& Taşkın, A. (2010). Effects of extremely low-frequency magnetic field on caspase activities and oxidative stress values in rat brain. Biological Trace Element Research, 138(1-3), 238-49.

Awad, S. M., \& Hassan, N. S. (2008). Health Risks of electromagnetic radiation from mobile phone on brain of rats. Journal of Applied Sciences Research, 4(12), 1994-2000.

Ayala, A., Muñoz, M. F., \& Argüelles, S. (2014). Lipid peroxidation: production, metabolism, and signaling mechanisms of malondialdehyde and 4-hydroxy-2-nonenal. Oxidative Medicine and Cellular Longevity, 2014(6), 360438. doi: $10.1155 / 2014 / 360438$

Ayata, A., Mollaoglu, H., Yilmaz, H. R., Akturk, O., Ozguner, F., \& Altuntas, I. (2004). Oxidative Stress-Mediated Skin Damage in an Experimental Mobile Phone Model Can Be Prevented by Melatonin. Journal of Dermatology, 31(11), 878-83.

Bediz, C. S., Baltaci, A. K., Mogulkoc, R., \& Öztekin, E. (2006). Zinc supplementation ameliorates electromagnetic fieldinduced lipid peroxidation in the rat brain. Tohoku Journal of Experimental Medicine, 208(2), 133-40.

Canseven, A. G., Coskun, S., \& Seyhan, N. (2008). Effects of various extremely low frequency magnetic fields on the free radical processes, natural antioxidant system and respiratory burst system activities in the heart and liver tissues. Indian Journal of Biochemistry \& Biophysics, 45(5), 326-31.
Consales, C., Merla, C., Marino, C., \& Benassi, B. (2012). Electromagnetic fields, oxidative stress, and neurodegeneration. International Journal of Cell Biology, 2012. doi: 10.1155/2012/683897

Coskun, S., Balabanll, B., Canseven, A., \& Seyhan, N. (2009). Effects of continuous and intermittent magnetic fields on oxidative parameters in vivo. Neurochemical Research, 34(2), 238-43.

Cui, K., Luo, X., Xu, K., \& Murthy, M. V. (2004). Role of oxidative stress in neurodegeneration: recent developments in assay methods for oxidative stress and nutraceutical antioxidants Progress in Neuro-Psychopharmacology and Biological Psychiatry, 28(5), 771-99.

Fang, Y. Z., Yang, S., \& Wu, G. (2002). Free radicals, antioxidants, and nutrition. Nutrition, 18(10), 872-79.

Fariss, M. W., \& Zhang, J. G. (2003). Vitamin E therapy in Parkinson's disease. Toxicology, 189(1), 129-46.

Fridovich, I. (1999). Fundamental aspects of reactive oxygen species, or what's the matter with oxygen? Annals of the New York Academy of Sciences, 893(1), 13-18.

Gilgun-Sherki, Y., Melamed, E., \& Offen, D. (2001). Oxidative stress induced-neurodegenerative diseases: the need for antioxidants that penetrate the blood brain barrier. Neuropharmacology, 40(8), 959-75.

Goraca, A., Ciejka, E., \& Piechota, A. (2010). Effects of extremely low frequency magnetic field on the parameters of oxidative stress in heart. Journal of Physiology and Pharmacology, 61(3), 333-38.

Gutteridge, J. M. (1995). Lipid peroxidation and antioxidants as biomarkers of tissue damage. Clinical Chemistry, 41(12), 1819 828.

Ilhan, A., Gurel, A., Armutcu, F., Kamisli, S., Iraz, M., Akyol, O., et al. (2004). Ginkgo biloba prevents mobile phone-induced oxidative stress in rat brain. Clinica Chimica Acta, 340(1), 15362

Irmak, M. K., Oztas, E., Yagmurca, M., Fadillioglu, E., \& Bakir B. (2003). Effects of electromagnetic radiation from a cellular telephone on epidermal Merkel cells. Journal of Cutaneous Pathology, 30(2), 135-38.

Jelenković, A., Janać, B., Pešić, V., Jovanović, D. M., Vasiljević, I., \& Prolić, Z. (2006). Effects of extremely low-frequency magnetic field in the brain of rats. Brain Research Bulletin, 68(5), 355-60.

Johansen, Ch. (2000). Exposure to electromagnetic fields and risk of central nervous system disease in utility workers. Epidemiology, 11(5), 539-43.

Kerman, M., \& Senol, N. (2012). Oxidative stress in hippocampus induced by $900 \mathrm{MHz}$ electromagnetic field emitting mobile phone: protection by melatonin. Biomedical Research, 23(1), 147-51.

Köylü H., Mollaoglu, H., Ozguner, F, Nazýroölu, M. \& Delibap, N. (2006). Melatonin modulates $900 \mathrm{MHz}$ microwaveinduced lipid peroxidation changes in rat brain. Toxicology and Industrial Health, 22(5), 211-16.

Lee, B. C., Johng, H. M., Lim, J. K., Jeong, J. H., Baik, K. Y., Nam T. J., et al. (2004). Effects of extremely low frequency magnetic field on the antioxidant defense system in mouse brain: a 
chemiluminescence study. Journal of Photochemistry and Photobiology B: Biology, 73(1), 43-48.

Mattson, M. P. (2004). Metal-Catalyzed Disruption of Membrane Protein and Lipid Signaling in the Pathogenesis of Neurodegenerative Disorders. Annals of the New York Academy of Sciences, 1012(1), 37-50.

McCord, J. M. (2000). The evolution of free radicals and oxidative stress. American Journal of Medicine, 108(8), 652-59.

Meral, I., Mert, H., Mert, N., Deger, Y., Yoruk, I., Yetkin, A., \& Keskin, S. (2007). Effects of 900-MHz electromagnetic field emitted from cellular phone on brain oxidative stress and some vitamin levels of guinea pigs. Brain Research, 1169, 12024. doi: 10.1016/j.brainres.2007.07.015

Mohammadnejad, D., Rad, J. S., Azami, A., \& Lotfi, A. (2011). Role of vitamin $\mathrm{E}$ in prevention of damages in the thymus induced by electromagnetic field: ultrastructural and light microscopic studies. Bulletin of the Veterinary Institute in Pulawy, 55, 111-15.

Moussa, S. A. (2009). Oxidative stress in rats exposed to microwave radiation. Romanian Journal of Biophysics, 19(2), 149-58.

Hamidian Jahromi, M. J., Kargar Jahromi, H., Keshavarz, M., Mozafar, A., Bathaee, S. A., \& Mahmoudi Teimourabad, S, (2014). Investigating 500 micro-tesla electromagnetic field effects on testicular tissue changes and testosteron in syrian mice race bulb/c. International Journal of Biology, Pharmacy and Allied Sciences, 3(3), 296-303.

Nazıroğlu, M. (2007). New molecular mechanisms on the activation of TRPM2 channels by oxidative stress and ADP-ribose. Neurochemical Research, 32(11), 1990-2001.

Oral, B., Guney, M., Ozguner, F., Karahan, N., Mungan, T., Comlekci, S., et al. (2006). Endometrial apoptosis induced by a 900-MHz mobile phone: preventive effects of vitamins $\mathrm{E}$ and C. Advances in Therapy, 23(6), 957-73.

Özmen, İ., Nazıroğlu, M., Alici, H. A., Şahin, F., Cengiz, M., \& Eren, I. (2007). Spinal morphine administration reduces the fatty acid contents in spinal cord and brain by increasing oxidative stress. Neurochemical Research, 32(1), 19-25.

Pezeshkian, M., Darbin, A., Rashidi, M. R., Vatankhah, A. M., Golmohammadi, Z., Afrasiabi, A., et al. (2011). The Effect of Atherogenic Diet with or without Enzyme Inhibitors on the Incidence and Progress of Atherosclerosis in Rabbits. Journal of Cardiovascular and Thoracic Research, 3(1), 7-10.

Rollwitz, J., Lupke, M., \& Simkó, M. (2004). Fifty-hertz magnetic fields induce free radical formation in mouse bone marrowderived promonocytes and macrophages. Biochimica et Biophysica Acta (BBA)-General Subjects, 1674(3), 231-38.

Salford, L. G., Brun, A. E., Eberhardt, J. L., Malmgren, L., \& Persson, B. R. (2003). Nerve cell damage in mammalian brain after exposure to microwaves from GSM mobile phones. Environmental Health Perspectives, 111(7), 881-83.

Salganik, R. I. (2001). The benefits and hazards of antioxidants: controlling apoptosis and other protective mechanisms in cancer patients and the human population. Journal of the American College of Nutrition, 20(5), 464-72.

Savitz, D. A., Checkoway, H., \& Loomis, D. P. (1998). Magnetic field exposure and neurodegenerative disease mortality among electric utility workers. Epidemiology, 9(4), 398-404.
Somi, M. H., Hajipour, B., Asl, N. A., Estakhri, R., Azar, A N., Zade, M. N., et al. (2009). Pioglitazone attenuates ischemia/reperfusion-induced liver injury in rats. Transplantation Proceedings, 41(10), 4105-109. doi: 10.1016/j.transproceed.2009.09.075

Tappel, A. L. (1972). Vitamin E and free radical peroxidation of lipids. Annals of the New York Academy of Sciences, 203(1), 12-28.

Van Acker, S. A., Koymans, L. M., \& Bast, A. (1993). Molecular pharmacology of vitamin E: structural aspects of antioxidant activity. Free Radical Biology and Medicine, 15(3), 311-28.

Yilmaz, H. R., Uz, E., Yucel, N., Altuntas, I., \& Ozcelik, N. (2004) Protective effect of caffeic acid phenethyl ester (CAPE) on lipid peroxidation and antioxidant enzymes in diabetic rat liver. Journal of Biochemical and Molecular Toxicology, 18(4), 234-38.

Yokus, B., Cakir, D. U., Akdag, M. Z., Sert, C., \& Mete, N. (2005). Oxidative DNA damage in rats exposed to extremely low frequency electro magnetic fields. Free Radical Research, 39(3), $317-23$.

Zhang, J., Zhang, Y. H., Jiang, R. P, Lian, Z. S, Wang, H., Luo, R.,et al. (2011). Protective effects of vitamin E against electromagnetic radiation from cell phones in pregnant and fetal rats brain tissues. Journal of Shandong University (Health Sciences), 9, 9-14. 